\title{
Experimental analysis of out-of-plane structural vibrations of two-wheeled vehicles
}

\author{
V. Cossalter*, A. Doria, R. Basso and D. Fabris \\ Department of Mechanical Engineering, University of Padova, Via Venezia 1, 35131 Padova, Italy
}

\begin{abstract}
An analysis of the handling and stability of two-wheeled vehicles depends on structural flexibility. This paper deals with laboratory experimentation carried out to identify the vehicle's structural modes of vibration. The tests were carried out in stationary conditions considering several combinations of constraints.

Although the testing conditions did not correspond completely to actual road conditions, information about the influence of structural modes on weave and wobble modes was obtained. An analysis of the Frequency Response Functions obtained from several kinds of tests made it possible to establish the best testing conditions to obtain the desired information.

Experimental results regarding a super-sport motorcycle and a maxi-scooter are presented and discussed in this paper.
\end{abstract}

\section{Introduction}

At present the dynamic behaviour of two-wheeled vehicles is studied using powerful multi-body codes (e.g. [1, 9]), which usually assume rigid behaviour of the front and rear frames, and take into account tire-road interaction by means of specific models (e.g. Paceika's Magic Formula [10]). Stability analysis is carried out linearizing the equations and studying small perturbations from straight running or steady cornering motion $[2,4]$.

Few researchers have analysed the effect of structural flexibility on the dynamic behaviour of two-wheeled vehicles [5,11,16,17]. In 1980 Sharp and Alstead [14] developed multi-body models taking into account in a simple way the lateral flexibility and torsional compliance of the front fork, and the twisting flexibility around an axis perpendicular to the steering head. They showed that large lateral flexibilities of the front fork have a significant influence on the stability of wobble and weave, whereas twisting flexibility around an axis perpendicular to the steering head has a large influence on wobble stability. Wobble is an oscillating mode $(8-10 \mathrm{~Hz})$ of the running vehicle dominated by the rotation of the front assembly around the steering axis. Weave is an oscillating mode $(2-3 \mathrm{~Hz})$ of the running vehicle that involves the whole vehicle and shows the largest displacements in the rear assembly.

The most recent multi-body models developed by Sharp $[13,15]$ take into account frame twisting stiffness as well, and their numerical simulations highlight some effects of this parameter on wobble instability. In [6] the response of the steering angle to road disturbances during cornering was studied and the transfer functions were evaluated numerically in the low frequency range $(<16 \mathrm{~Hz})$.

In recent years some significant changes have appeared in the field of two-wheeled vehicles: new structural materials, new frame design concepts, new vehicles (e.g. maxi-scooters with large displacement engines). Hence, the problem of the influence of structural vibrations of the frame on the features of stability and handling continues to be relevant.

The aim of this study was to tackle this subject experimentally, by developing proper modal analysis techniques in order to identify, with laboratory tests, the structural modes of vibration that may interfere with stability and handling of two-wheeled vehicles.

\footnotetext{
*Corresponding author. E-mail: vittore.cossalter@unipd.it.
} 


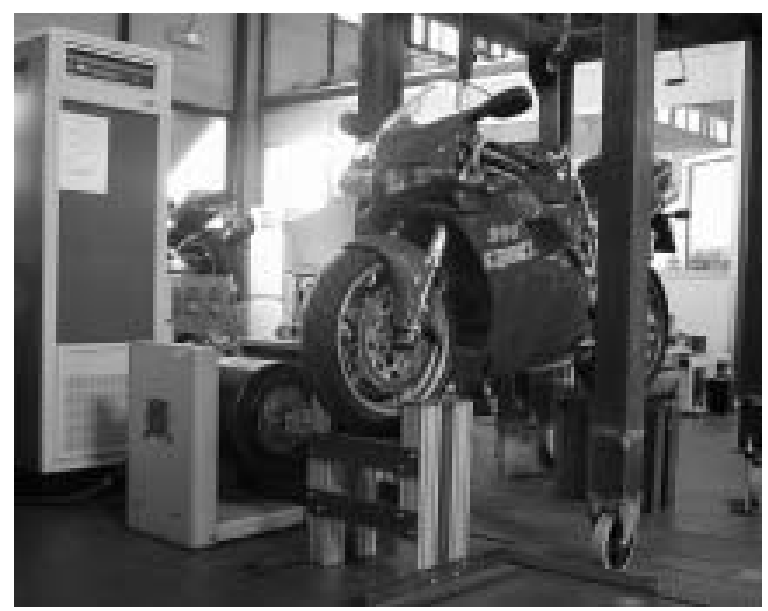

Fig. 1. Testing equipment.

\section{Testing for the modal analysis of two-wheeled vehicles}

A Single-Input Multi-Output (SIMO) technique was used, since the main purpose is identification of out-of-plane structural modes. The excitation was applied to a point of the vehicle and accelerations were measured in several significant points. These points constituted the mesh of the experimental model and their location on the vehicle was evenly distributed. Since the information about handling was of great importance, one measurement point was located on the handlebars.

In a previous work [2] impulsive excitation was used in order to identify the lower modes of vibration of two super-sport motorcycles and quite good results were achieved. In order to overcome the limitations of impulsive excitation (e.g. low coherence), in this study the excitation was carried out using an electro-dynamic shaker.

\subsection{Experimental apparatus}

Figure 1 shows the experimental equipment. The two-wheeled vehicle was suspended from a support by very soft springs, which guaranteed the vehicle equilibrium and insulated it from support vibrations.

The front (or rear) tire of the vehicle was supported by a vibrating table, whose motion was imposed by the shaker. The table was covered with high friction material to simulate road grip. A load cell was mounted between the shaker head and the vibrating table. In these conditions, the excitation took place in the lateral direction, called the y-direction according to the standard [12] for the reference frames of motorcycles (longitudinal and vertical directions are called $x$ - and $z$-directions respectively).

The motion of the vibrating table was set as an up/down sine sweep with logarithmic rate. Acceleration amplitude was set to a constant value (e.g. 1 or $2 \mathrm{~m} / \mathrm{s}^{2}$ ) and frequency ranged from 10 to $50 \mathrm{~Hz}$.

Since the shaker generated higher harmonics when it worked in the lower frequency range, the total frequency range was split into a low (below 15-18 Hz) and a high frequency range (up to $50 \mathrm{~Hz}$ ).

The tests were conducted without a rider and the front assembly was free to rotate about the steer axis.

In the framework of this research two vehicles were tested: a super-sport motorcycle (vehicle A) equipped with a tubular steel frame and a single-sided swinging arm and a maxi-scooter (vehicle B) equipped with a 500 cc engine.

\subsection{Constraint conditions}

The modal characteristics of a two-wheeled vehicle should be identified by testing the vehicle when it is running on the road. Since these tests are very difficult to carry out, experimental modal analysis has to be carried out in laboratory setting the constraint conditions to be as similar as possible to those experienced when the motorcycle is running on the road. 
When a wheel rolls and slips laterally, tire interaction with the road in the lateral $(y)$ direction can be approximated with a model that includes an equivalent damper in series with tire lateral stiffness $k_{y}[4,7]$. The damping coefficient of this damper $(c)$ is roughly inversely proportional to forward speed $V$ and proportional to cornering stiffness $K_{\lambda}$ (the slope of the curve of the lateral force versus side-slip angle when side-slip is zero). Therefore, the mechanical impedance $\left(Z_{y}\right)$ of the two-wheeled vehicle at the interface with the road is given by equation:

$$
Z_{y}=\frac{k_{y} c}{c i \omega+k_{y}}
$$

where $\omega$ is the angular frequency and $c=K_{\lambda} / V$. Equation (1) shows that $Z_{y}$ tends to zero for very high speeds and $k_{y} / i \omega$ for very low speeds.

In the vertical direction there is no slip and the contact between the tire and the road can be modelled by means of a spring simulating the radial stiffness of the tire $k_{z}$; the mechanical impedance is $k_{z} / i \omega$.

During laboratory tests on the stationary vehicle, if a wheel is in contact with a stationary surface the mechanical impedance is $k_{y} / i \omega$ in the lateral direction and $k_{z} / i \omega$ in the vertical direction. If a wheel is in contact with a vibrating table, the mechanical impedance in the lateral direction is again $k_{y} / i \omega$, but it is defined as the ratio between the lateral force and the relative velocity between the wheel hub and the table.

Since neither the tests in the free conditions $\left(Z_{y}=0\right)$, nor the tests in the contact conditions $\left(Z_{y}=k_{y} / i \omega\right)$ correctly simulate actual road-tire interaction, some differences between the low frequency modes identified in the laboratory and the actual modes on the road can be expected.

\subsection{Modes dominated by tire compliance}

During laboratory tests with both wheels in contact with high friction surfaces, low frequency modes dominated by tire compliance may be excited. In these modes, which are called "Tire Dominated Modes" (TDMs), the front and rear assemblies behave essentially as rigid bodies.

The TDMs can be studied according to the motorcycle model in Fig. 2. This model is derived from the one presented in [4] for the simplified analysis of weave and wobble. It takes into account only out-of-plane displacements and has three degrees of freedom: the lateral displacement of the steering axis $(w)$, the rotation of the front assembly $\left(\theta_{f}\right)$ and the rotation of the rear assembly $\left(\theta_{r}\right)$. The contact patches of the tires are assumed to adhere to the road and the lateral stiffness of the tires is simulated by means of springs. The equation of free undamped vibrations is:

$$
\left[\begin{array}{ccc}
M_{f}+M_{r} & -M_{r} l_{1} & M_{f} b_{f} \\
-M_{r} l_{1} & M_{r} l_{1}^{2}+I_{r} & 0 \\
M_{f} b_{f} & 0 & M_{f} b_{f}^{2}+I_{f}
\end{array}\right]\left\{\begin{array}{c}
\ddot{w} \\
\ddot{\theta}_{r} \\
\ddot{\theta}_{f}
\end{array}\right\}+\left[\begin{array}{ccc}
k_{f}+k_{r} & -l k_{r} & -a_{n} k_{f} \\
-l k_{r} & l^{2} k_{r} & 0 \\
-a_{n} k_{f} & 0 & a_{n}^{2} k_{f}
\end{array}\right]\left\{\begin{array}{l}
w \\
\theta_{r} \\
\theta_{f}
\end{array}\right\}=\left\{\begin{array}{l}
0 \\
0 \\
0
\end{array}\right\}
$$

Where $M_{r}$ and $M_{f}$ are rear and front masses, $I_{r}$ and $I_{f}$ rear and front moments of inertia about the steering axis and $k_{r}$ and $k_{f}$ rear and front tire lateral stiffness. On the one hand, the modal shapes of TDMs are similar to weave and wobble ones (which are essentially rotations of the rear and front frame around the steering axis). On the other hand, TDM frequencies, which depend only on the inertial properties of the vehicle and the stiffness of the tire carcass, range from 10 to $20 \mathrm{~Hz}$ and are rather different from weave and wobble ones, which depend on the gyroscopic effect and the slip properties of tires as well.

\section{Experimental results - the effect of contact}

Some tests were carried out on vehicle A (super-sport) in order to highlight the effect of the wheels' contact on the measured Frequency Response Functions (FRFs) and to find the best testing conditions. Four different conditions were considered:

\begin{tabular}{ll} 
Acronym & \multicolumn{1}{c}{ Rear wheel } \\
RE-FF & Excited by the vibrating table \\
RE-FC & Excited by the vibrating table \\
RF-FE & Free condition \\
RC-FE & contact with a stationary plane
\end{tabular}

Front wheel

Free condition

Contact with a stationary plane

Excited by the vibrating table

Excited by the vibrating table 


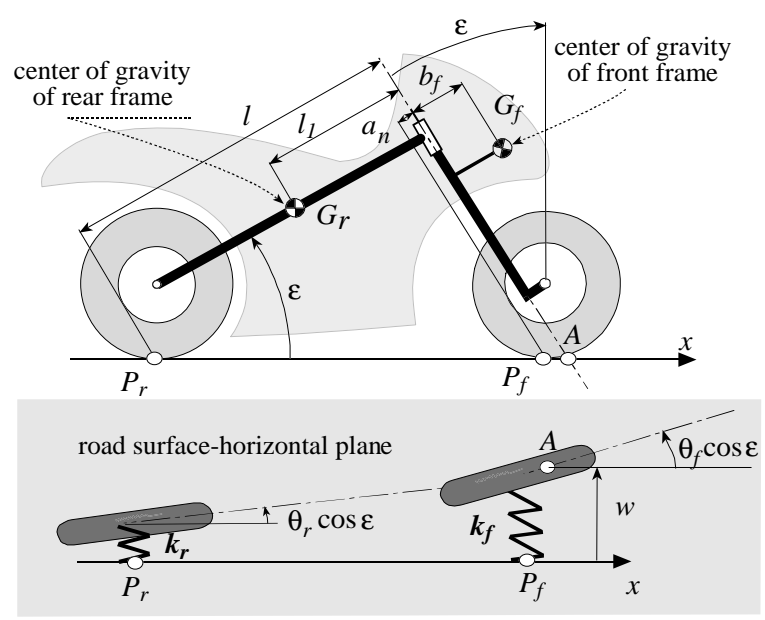

Fig. 2. Model for the evaluation of Tire Dominated Modes.

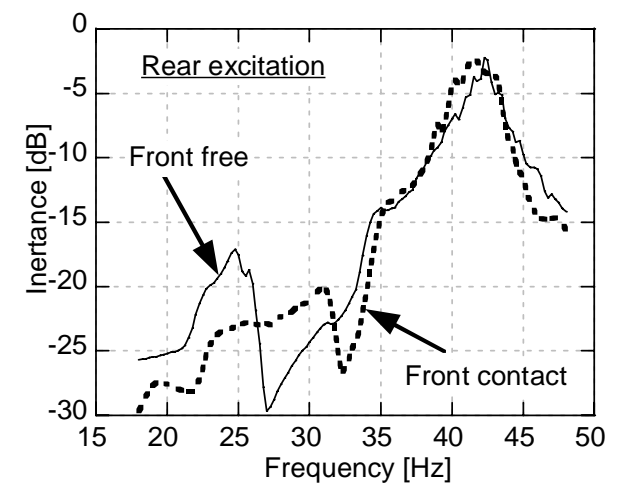

Fig. 3. Inertance of measurement point $\mathrm{P}$ on the handlebars, lateral direction $(y)$.

Owing to the presence of the elastic suspension the total tire load was about $80 \%$ of the vehicle's weight.

A typical example of experimental results obtained with rear excitation in the lateral direction $(y)$ is presented in Fig. 3, which shows the FRF (inertance) in the lateral direction $(y)$ of a point on the handlebars (point $\mathrm{P}$, see Fig. 6). With the front wheel in the free condition, the largest amplitudes are in the range $33-45 \mathrm{~Hz}$ moreover, there is a peak at about $25 \mathrm{~Hz}$. The introduction of the contact between the front tire and a stationary surface does not modify the response above $33 \mathrm{~Hz}$, whereas it causes significant changes below $33 \mathrm{~Hz}$. Hence, on the one hand, the high frequency modes greatly depend on structural flexibility and are slightly influenced by tire contact; on the other hand, the mode at $25 \mathrm{~Hz}$ is influenced by tire contact.

Figure 4 refers to results obtained in the same testing conditions, but the inertance is measured in the longitudinal direction $(x)$. The response in this direction is sensitive to the handlebars' rotation around the steering axis and, hence to TDMs. The frequency response below $18 \mathrm{~Hz}$ is also considered. The inertance levels are lower than in the previous case because the directions of measurement $(x)$ and excitation $(y)$ are perpendicular.

With the front contact there are significant peaks in the range $10-20 \mathrm{~Hz}$ that may be related to TDMs.

Hence, testing condition RE-FC causes the excitation of TDM modes too; the FRFs measured at the other points confirm this statement.

A typical example of experimental results obtained with front excitation in the lateral direction $(y)$ is presented in Fig. 5, which shows the FRF (inertance) in the lateral direction of point $\mathrm{P}$ on the handlebars.

The shape of the FRF is different from the one in Fig. 3 (rear excitation); the main peak is at about $25 \mathrm{~Hz}$ and small peaks appear in the range 30-40 Hz. Moreover, the presence of the rear contact has a limited influence on the FRF and causes a small increment in the frequency of the first peak, due to the increased stiffness. 


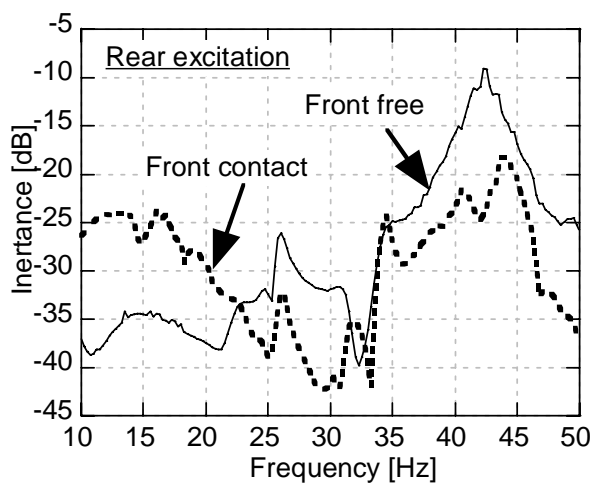

Fig. 4. Inertance of measurement point $\mathrm{P}$ on the handlebars, longitudinal direction $(x)$.

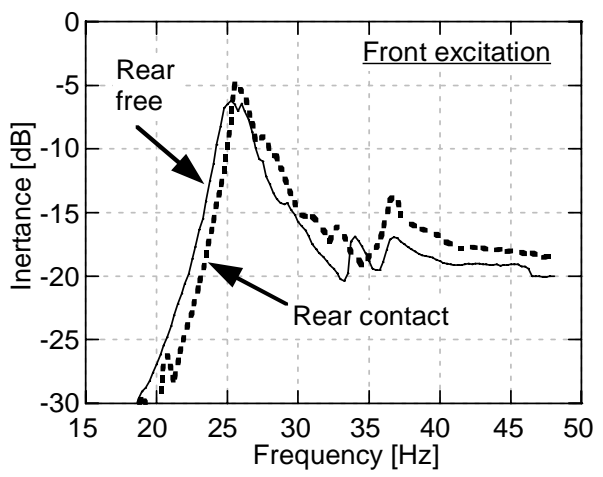

Fig. 5. Inertance of measurement point $\mathrm{P}$ on the handlebars, lateral direction $(y)$.

Some preliminary identification tests were carried out. On the one hand, the FRFs measured with front excitation made it possible to clearly identify the $25 \mathrm{~Hz}$ mode, which has an anti-node near the excitation point. However, the large excitation of this mode masked the other modes and made it difficult to identify them.

On the other hand, the FRFs measured with rear excitation made a satisfactory identification of the $25 \mathrm{~Hz}$ mode and the best identification of the other structural modes possible. TDMs were identified with rear excitation and front contact.

\section{Experimental results - the modes of vibration of a super-sport motorcycle}

In order to highlight the influence of nonlinearities, some preliminary tests were carried out on the most uncoupled modes by means of the single-FRF methods included in the software ICATS $\subset$. When different series of points on either sides of the resonance peak, which correspond to different amplitude levels, were used to identify the modal parameters, only small shifts (1-2\%) in the natural frequencies were found. Then, the modal parameters (natural frequencies, hysteretic damping, modal shapes) were identified from the measured inertances ( 96 records) by means of the multi-FRF methods [8] included in the software ICATS C). These methods are faster than single-FRF methods and make it possible to identify close modes. The best results were achieved by means of the Global Rational Fraction method [8]. Moreover, the results were validated through the Reciprocal Vector method.

In the figures below, the identified modes are represented in a plane perpendicular to the steering axis and the dashed line represents the zero deflection condition.

Testing condition RE-FC made it possible to identify two TDMs at 15.6 and $17.9 \mathrm{~Hz}$. Figure 6 shows that the rigid rotation of the front assembly around the steering axis dominates the first TDM. Figure 7 shows that in the second TDM, the rigid rotation of the front assembly is in opposition to the rotation of the rear assembly. 


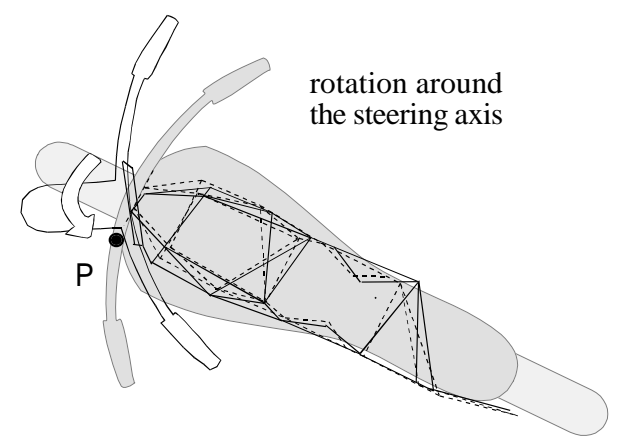

Fig. 6. Vehicle A, TDM, frequency $15.6 \mathrm{~Hz}$, hysteretic damping $9.5 \%$.

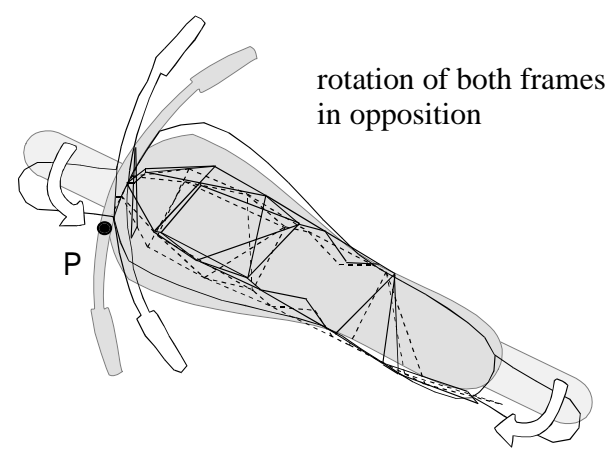

Fig. 7. Vehicle A, TDM, frequency $17.9 \mathrm{~Hz}$, hysteretic damping $8.4 \%$.

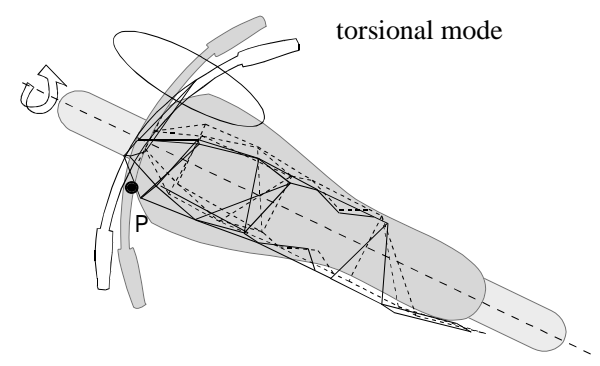

Fig. 8. Vehicle A, torsional mode, frequency $26.8 \mathrm{~Hz}$, hysteretic damping 5.0\%.

In every testing condition a structural mode dominated by the torsion of the rear frame near the steering head was identified. Torsional deformation causes a large lateral displacement of the fork.

The natural frequency and hysteretic damping of the torsional mode vary slightly according to the testing conditions, the modal shape appears to be clearer in the RC-FE testing condition, as shown in Fig. 8.

Figures 9 and 10 show the higher order modes (testing condition RE-FC). The mode at $34.8 \mathrm{~Hz}$ shows flexural deformation in the plane perpendicular to the steering axis. The lateral displacement of the front wheel is small, whereas large lateral displacements take place in the rear frame and in the swinging arm; all these lateral displacements have the same direction.

The mode at $40.9 \mathrm{~Hz}$ also shows flexural deformation in the plane perpendicular to the steering axis. The lateral displacement of the front wheel is small. Small lateral displacements also take place in the central part of the rear frame, which is a nodal zone. The lateral displacements of the rear frame near the steering head and of the swinging arm have opposite directions.

The comparison between a measured FRF and the one reconstructed by means of the identified modes is an index 


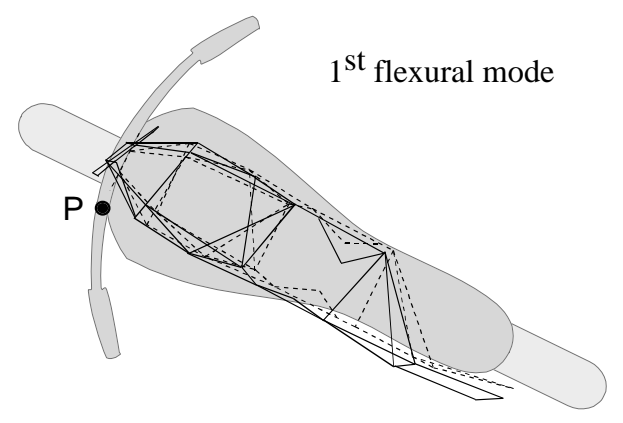

Fig. 9. Vehicle A, flexural mode, frequency $34.8 \mathrm{~Hz}$, hysteretic damping 7.2\%.

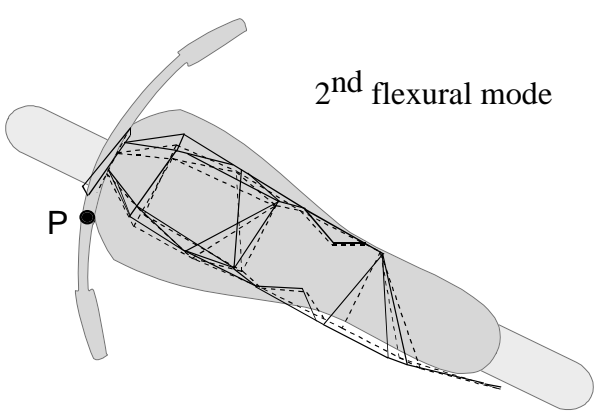

Fig. 10. Vehicle A, flexural mode, frequency $40.9 \mathrm{~Hz}$, hysteretic damping $6.9 \%$.

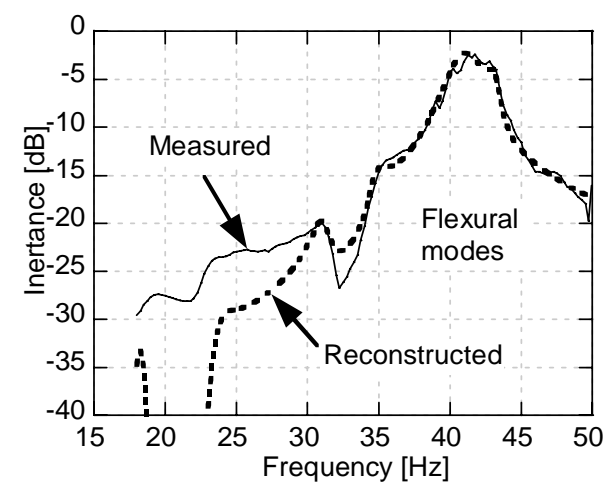

Fig. 11. RE-FC, FRF of point $\mathrm{P}$ on the handlebars.

of the quality of identification. Figures 11 and 12 clearly show that in testing condition RE-FC there is a satisfactory reconstruction of flexural modes $(34.8$ and $40.9 \mathrm{~Hz})$, whereas in testing condition RC-FE there is a satisfactory reconstruction of the torsional mode (natural frequency $26.8 \mathrm{~Hz}$ ).

\section{Experimental results - the modes of vibration of a maxi-scooter}

The maxi-scooter was tested in the RE-FF condition and the modal parameters were identified from 48 measured inertances using the above-cited multi-FRF methods.

The first structural mode has a natural frequency equal to $16.9 \mathrm{~Hz}$ and is dominated by torsional deformation. Figure 13 (in which the dashed line represents the zero deflection condition) shows the modal shape. The front frame, consisting of the front wheel and fork, rotates anti-clockwise with respect to axis $x$ (which is aligned with 


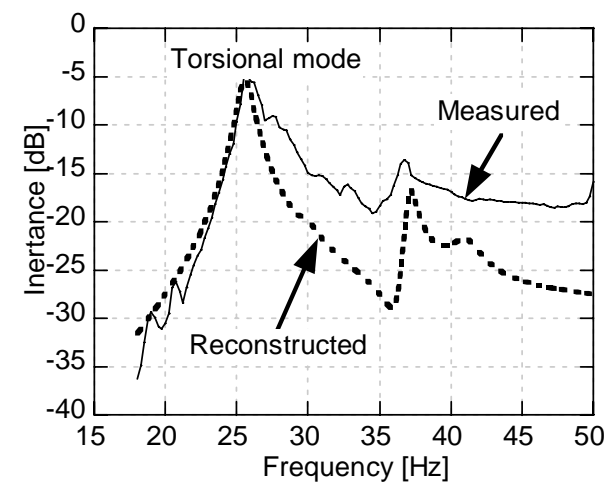

Fig. 12. RC-FE, FRF of point $\mathrm{P}$ on the handlebars.

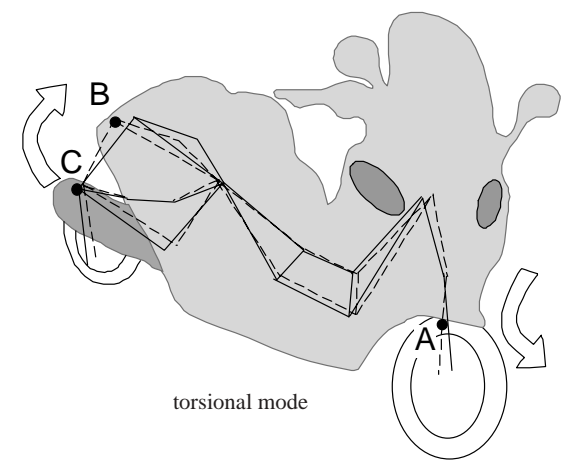

Fig. 13. Vehicle B, torsional mode, frequency $16.9 \mathrm{~Hz}$, hysteretic damping $4.8 \%$.

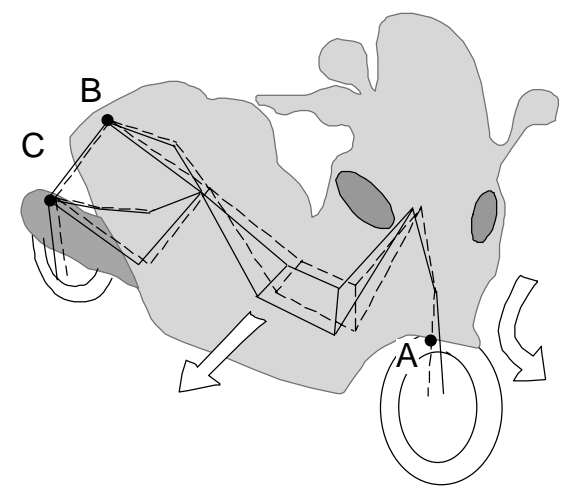

Fig. 14. Vehicle B, structural mode, frequency $19.7 \mathrm{~Hz}$, hysteretic damping $3.2 \%$.

the forward motion direction). The central part of the rear frame near the engine's attachment point is a nodal zone. The part of the rear frame beneath the saddle and the engine rotate clockwise with respect to axis $x$.

The second mode $(19.7 \mathrm{~Hz})$, which is represented in figure 14, shows a rigid lateral displacement of the rear frame and a rotation of the front frame about an axis perpendicular to the steering head that moves the front wheel laterally; the displacements of the rear frame and the front wheel have opposite directions.

The last identified mode $(24.7 \mathrm{~Hz})$ shows a combination of flexural and torsional deformation (Fig. 15).

In order to evaluate the quality of the identification, the reconstructed FRFs were compared with the measured ones. Figure 16, 17 and 18 deal with the FRFs (inertances) in the lateral direction ( $y$ ) of the mesh points located in the front fork (point A), the rear frame beneath the saddle (point B) and in the engine case (point C). 




Fig. 15. Vehicle B, structural mode, frequency $24.7 \mathrm{~Hz}$, hysteretic damping $2.9 \%$.

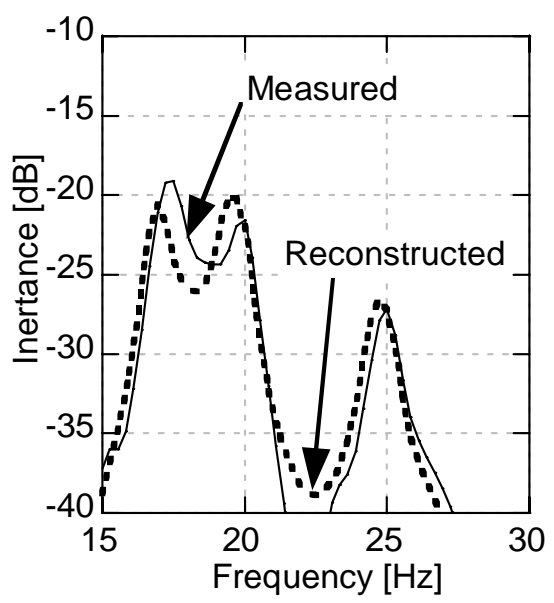

Fig. 16. Vehicle B, RE-FF, FRF of point A.

The reconstructed FRFs appear close to the experimental data in the range $15-30 \mathrm{~Hz}$, this result confirms the validity of the identified modes.

Some preliminary shaker tests carried out on another scooter (equipped with a $150 \mathrm{cc}$ engine) made it possible to identify three structural modes at $12.9,19.7$ and $28.7 \mathrm{~Hz}$. It is worth highlighting that low values of the natural frequencies of structural modes may influence the stability and handling of two-wheeled vehicles.

\section{Conclusions}

Front excitation and rear contact made possible the best identification of torsional modes, whereas rear excitation and front contact led to the best identification of structural modes in general.

The natural frequency of the first structural mode of the super-sport motorcycle is significantly higher than the frequencies typical of wobble phenomena, hence structural compliance has a small influence on stability. Impulsive modal testing carried out on another motorcycle of the same class [2] led to similar results, hence, the high frequency of the structural modes seems to be a feature of modern high performance motorcycles.

The first structural mode of the tested motorcycle is dominated by torsional deformation near the steering head. Therefore, the introduction of a torsional compliance about an axis perpendicular to the steering head seems to be suitable for a detailed numerical analysis of stability and handling.

The tests on the scooters showed that the first structural mode occurs at about $13-17 \mathrm{~Hz}$, hence, structural compliance may have a significant influence on the stability and handling properties of this type of vehicle. It's worth 


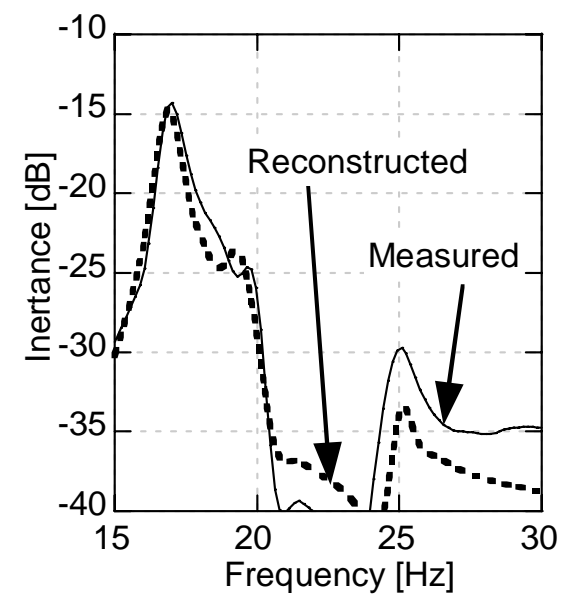

Fig. 17. Vehicle B, RE-FF, FRF of point B.

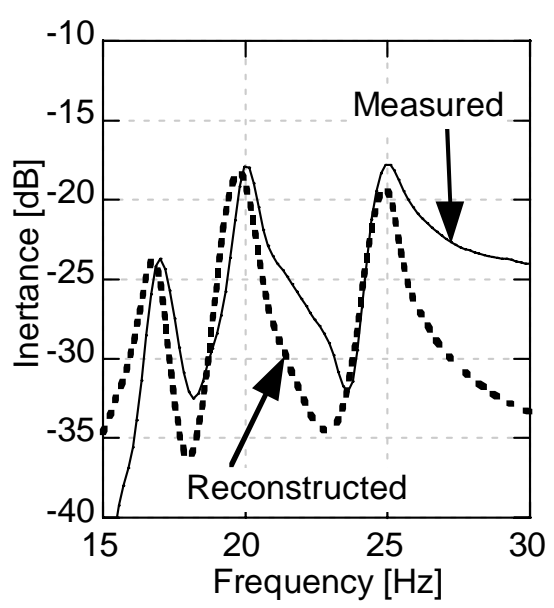

Fig. 18. Vehicle B, RE-FF, FRF of point C.

pointing out that the structural modes of a maxi-scooter can be excited by wheel unbalance, taking into account the small value of the rolling radius and the maximum speed of these vehicles, which is about $100-150 \mathrm{~km} / \mathrm{h}$.

\section{References}

[1] V. Cossalter and R. Lot, A motorcycle multi-body model for real time simulation based on the natural coordinates approach, Vehicle System Dynamics 37(6) (2002), 423-447.

[2] V. Cossalter, A. Doria and L. Mitolo, Inertial and modal properties of racing motorcycles, in: Proc. of Motorsports Engineering Conference \& Exhibition, Indianapolis, Indiana, USA, 2002, SAE Paper Number 02MSEC-5, pp. 605-612.

[3] V. Cossalter, R. Lot and F. Maggio, The influence of Tire Properties on the Stability of a Motorcycle in Straight Running and Curves, in Proc, of Automotive Dynamics \& Stability Conference, Detroit, Michigan, USA, 2002, SAE Paper Number 02ADSC-68.

[4] V. Cossalter, Motorcycle dynamics, Race Dynamics, Greendale WI, 2002.

[5] T. Hikichi and K. Takagi, Dynamic characteristics of a motorcycle with a single-side supported swinging arm, SAE paper 905214, 1990, pp. 721-731.

[6] D.J.N. Limebeer, R.S. Sharp and S. Evangelou, Motorcycle steering oscillations due to road profiling, Journal of Applied Mechanics, Transactions ASME 69(6) (2002), 724-739.

[7] R. Lot, A motorcycle tire model for dynamic simulations: theoretical and experimental aspects, in: Meccanica, to appear, 2003

[8] N.M.M. Maia and J.M. Montalvao e Silva, Theoretical and experimental modal analysis, John Wiley \& Sons Inc, New York, 1997. 
[9] L. Mitolo, R. Berritta and S. Garbin, Virtual prototyping of motorcycles with LMS.DADS and MSC.VisualNASTRAN multibody codes: evaluation of performances in typical manoeuvres, in: Proc. of 7th Int. Conf. "High-tech cars and engines", Modena, Italy, 31 May-1 June, 2001.

[10] H.B. Pacejka, Tyre and Vehicle Dynamics, Butterworth, Oxford, 2002.

[11] G.E. Roe and T.E. Thorpe, The influence of frame structure on the dynamics of motorcycle stability, SAE paper 891772, 1989, pp. 13191330 .

[12] SAE, Vehicle Dynamics Terminology, SAE Standard J670, 1976.

[13] R.S. Sharp, Stability, control and steering responses of motorcycles, Vehicle System Dynamics 35(4-5) (2001), 291-318.

[14] R.S. Sharp and C.J. Alstead, The influence of structural flexibilities on the straight-running stability of motorcycles, Vehicle System Dynamics 9 (1980), 327-357.

[15] R.S. Sharp and D.J.N. Limebeer, A Motorcycle model for stability and control analysis, Multibody System Dynamics (6) (2001), $123-142$.

[16] P.T.J. Spierings, The effect of lateral front fork flexibility on the vibrational modes of straight-running single-track vehicles, Vehicle System Dynamics 10 (1981), 21-35.

[17] M.K. Verma, R.A. Scott and L. Segel, Effect of frame compliance on the lateral dynamics of motorcycles, Vehicle System Dynamics $\mathbf{9}$ (1980), 181-206. 

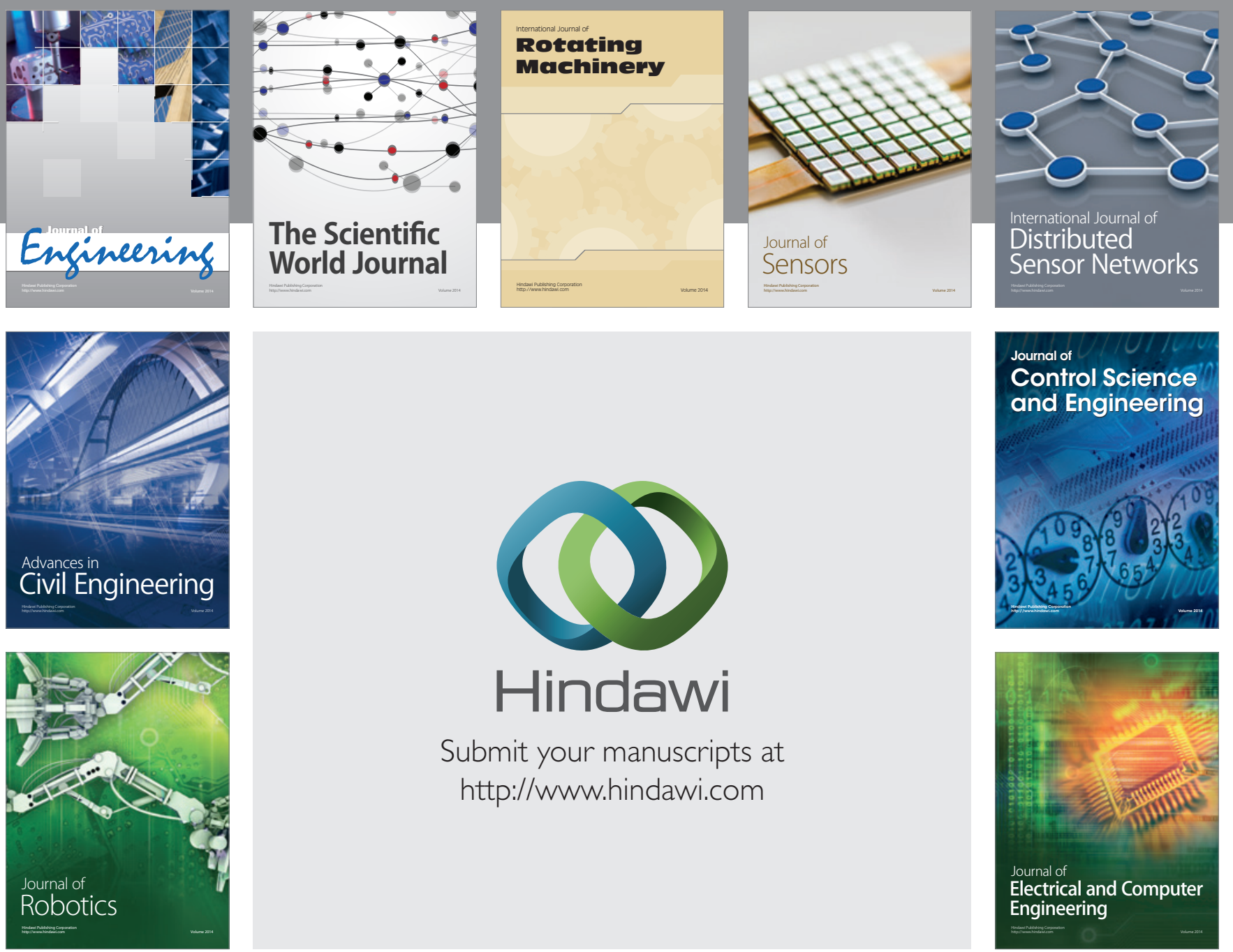

Submit your manuscripts at

http://www.hindawi.com
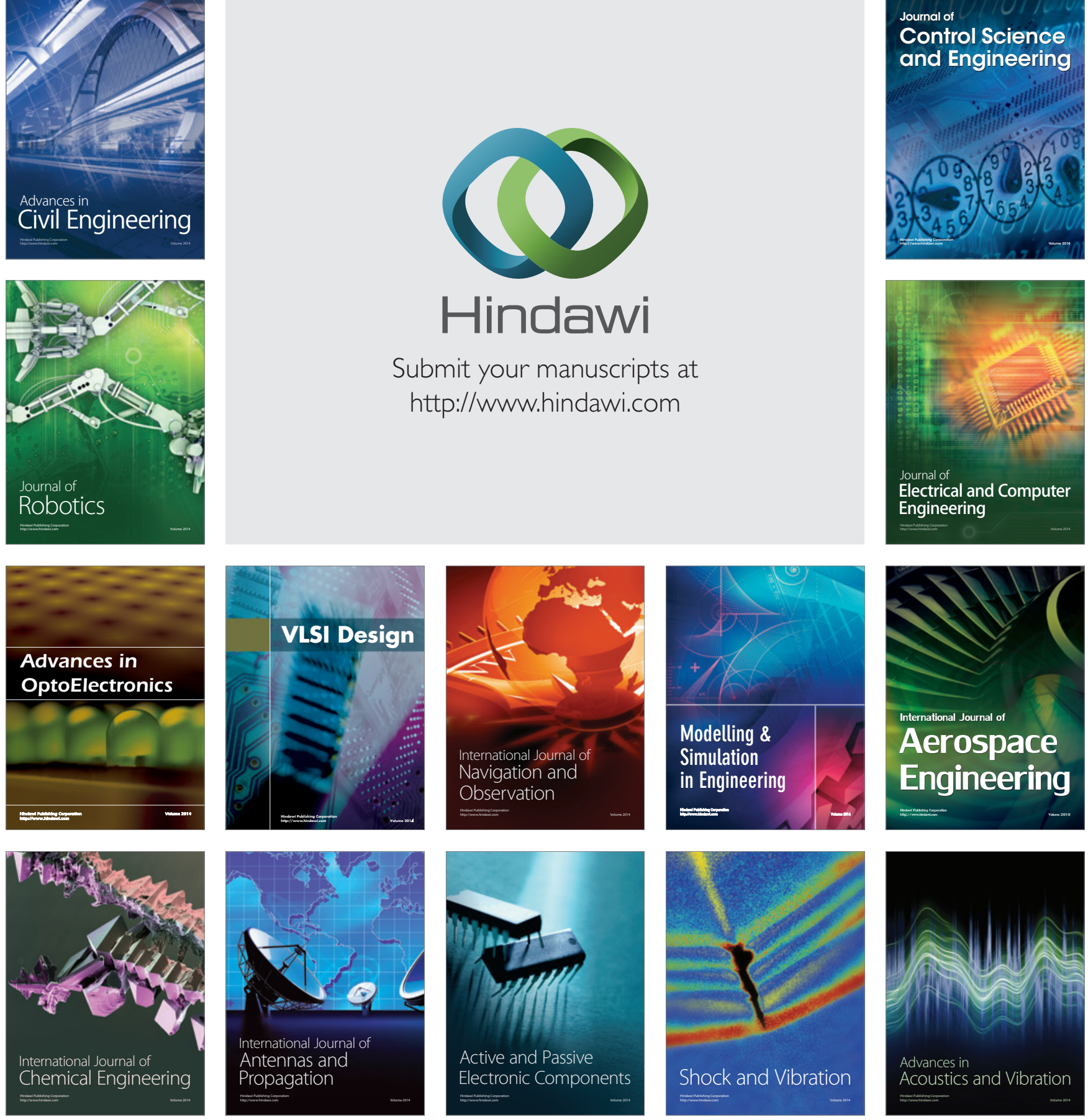